\title{
Quasar Spectroscopy with FLAIR
}

\author{
Quentin A. Parker ${ }^{1}$ \\ Anglo-Australian Observatory, Coonabarabran, NSW 2357, Australia \\ R.G. Clowes \\ University of Central Lancashire, Preston, England \\ F.G. Watson \\ Royal Greenwich Observatory, Cambridge, England
}

\begin{abstract}
We demonstrate that the multi-object fibre-spectroscopy system 'FLAIR' on the UKST can be used to observe efficiently and effectively the brighter $(B<18.5)$ high-grade quasar candidates identified from UKST low-dispersion objective-prism plates. The quasar candidates were selected objectively by the application of AQD (automatic quasar detection) to prism plates that have been digitised using the COSMOS measuring machine. Thirteen high-grade quasar candidates with $B \leq 18.5$ from a prism plate of ESO/SERC survey field 296 were observed with a 35 -fibre version of FLAIR. From a total observing time of only 3 hours at a resolution of $8.4 \AA /$ pixel six candidates were confirmed as quasars and four were identified as stars. The planned upgrade to a thinned, back-illuminated CCD in 1994 should yield a 3-fold increase in sensitivity and further enhance the capabilities of FLAIR for performing useful spectroscopic observations of quasars of moderate faintness over large areas of sky.
\end{abstract}

\section{Introduction}

We present some early results on the use of the unique FLAIR multi-object, fibre-optic spectroscopy system on the UK Schmidt Telescope (UKST) to observe faint $B<18.5$ magnitude quasars. These observations represent the first serious attempt to use FLAIR to observe such faint objects and the results are presented here for the first time. FLAIR is in many ways an ideal choice for survey work on samples obtained from UKST plates as it can simultaneously observe many target objects over the full $40 \mathrm{deg}^{2}$ field of the UKST provided that adequate $S / \mathrm{N}$ can be achieved. For this project quasar candidates were selected objectively from UKST low dispersion $(2480 \AA / \mathrm{mm}$ at $H \gamma)$ objective-prism plates using the automatic quasar detection software (AQD) developed by Clowes (Clowes 1986).

\footnotetext{
${ }^{1}$ Currently on special leave from the Royal Observatory Edinburgh, UK
} 
Spectroscopic follow-up observations of the AQD candidates is necessary to confirm both the quasar identification and the redshift. Such follow-up may be enhanced by the use of FLAIR to observe the brighter candidates, with allocations of time on $4 \mathrm{~m}$ telescopes devoted to the fainter candidates. With $4 \mathrm{~m}$ telescopes and only a 40 arcmin field of view, the surface density of candidates is often such that multi-object spectroscopy is less efficient than single-object spectroscopy.

FLAIR was considered as a possible option to assist AQD spectroscopic follow-up. This was because its field of view matches the original UKST plates from which the samples were obtained and because the relatively small aperture of the UKST $(1.2 \mathrm{~m})$ is partially offset by the exceedingly long dwell times that are possible. Numerous $3000 \mathrm{~s}$ exposures of a field can be obtained with the same fibre set-up. These can then be co-added to yield higher $\mathrm{S} / \mathrm{N}$ spectra. No other spectroscopic facility offers wide-field $\left(40 \mathrm{deg}^{2}\right)$ coverage with a simultaneous multi-object capability.

\section{AQD selection}

Firstly a suitable target sample was obtained using AQD. AQD is a software based technique which objectively applies controlled selection criteria to digitised low dispersion objective-prism spectra. The digitisation and plate scanning were performed by the COSMOS measuring machine (MacGillivray \& Stobie 1984). Rather than scanning the plates by eye the technique electronically sifts through the 60,000 or so objective-prism spectra detected on a typical UKST low-dispersion prism plate. Only candidate spectra that satisfy criteria specifically tailored to identify quasar-type spectral features are selected (e.g. presence of emission lines and/or UV excess). The original description of the AQD technique is given by Clowes et al. (1984) though the candidates selected for this study were obtained using an enhanced AQD process described by Clowes (1986). The technique has been shown to be highly successful at discovering quasars and was instrumental in the discovery of the third 'quasar superstructure' (Clowes \& Campusano 1991). These structures appear to indicate coherence on $\sim 100 h^{-1}$ Mpc scales.

AQD was applied to a good quality objective-prism plate of ESO/SERC survey field 296 (UKST plate UJ5406P, 1950 field-centre: $01^{h} 18^{m} 00^{s},-40^{\circ} 00^{\prime} 00^{\prime \prime}$ ) as part of a large AQD quasar survey over 7 contiguous UKST fields near the SGP (covering $\sim 200 \mathrm{deg}^{2}$ ). After rejection of obvious contaminants due to overlaps, a sample of 213 high grade AQD candidates was identified. High grade candidates are those for which both emission and ultraviolet excess were detected in the objective-prism spectra. The plate magnitude limit was $B \sim 20.5$, typical for the deepest prism plates.

\section{FLAIR observations}

The observations were made with the PANACHE development version of the FLAIR system as described by Watson (1988) during a FLAIR run in October 1988. This system had a modest $35 \times 100 \mu \mathrm{m}(6.7$ arcsec $)$ fibre feed. A selection of 14 of the brightest high-grade AQD candidates were chosen for observation 

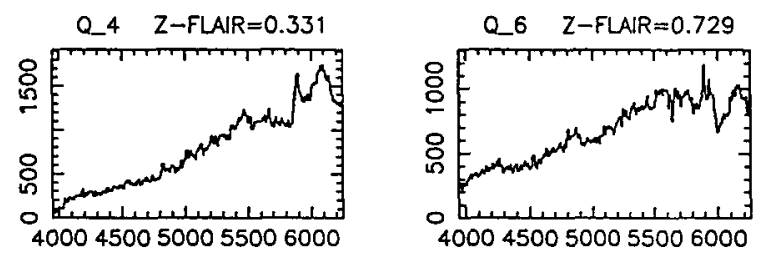

Q.7 Z-FLAR $=2.158$
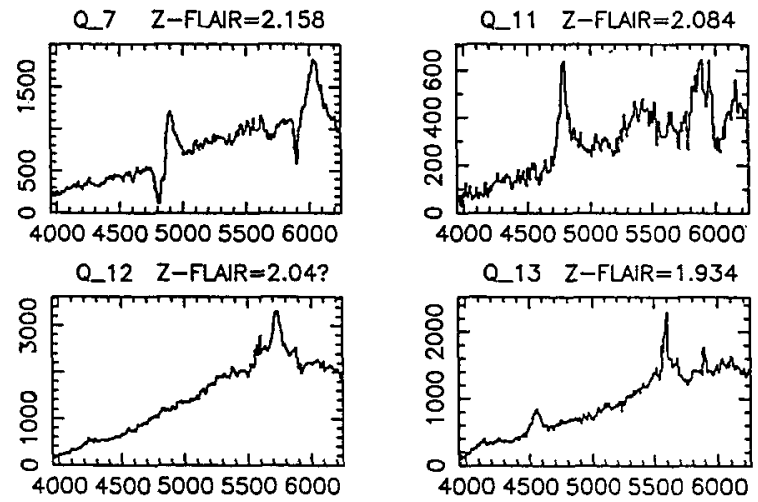

WATELENGTH $(A)$

Figure 1. A montage of the 6 AQD confirmed QSO FLAIR spectra
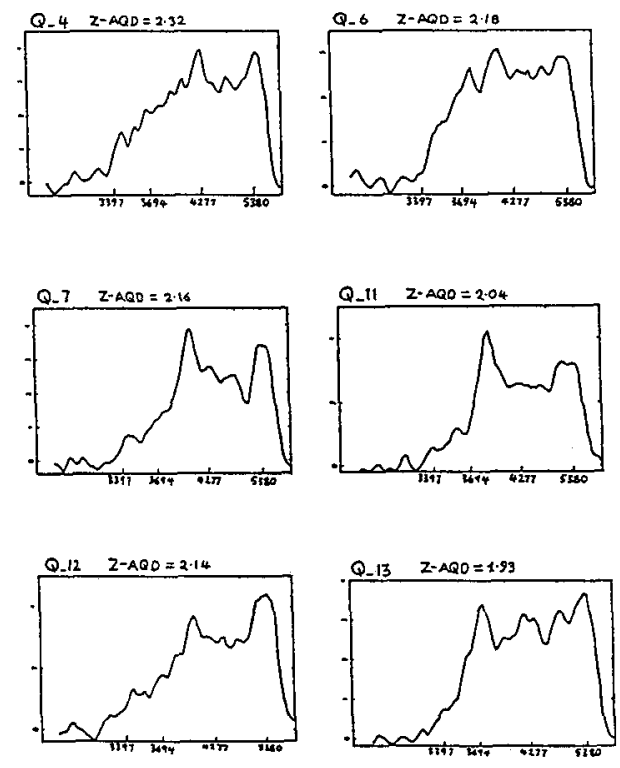

WAVELENGTH ( $\AA$ )

Figure 2. A montage of the corresponding objective-prism AQD spectra 
with the remaining fibres being dedicated to faint $(B \leq 17.8)$ IRAS galaxies. Five sky fibres were also assigned.

A $600 \mathrm{~V}$ grating was used with on-chip binning to provide $8.4 \AA /$ pixel resolution $(\sim 196 \AA / \mathrm{mm})$. Six separate 1800 second exposures were obtained, along with associated arc frames, biases, dome-flats etc. Arc frames were only required immediately before and after all the target field exposures had been taken. This is because the fibre-fed spectrograph sits on a vibrationally isolated optical bench on the dome floor. This gives an inherently very stable instrumental configuration with highly consistent wavelength stability between consecutive exposures (see Parker \& Watson 1990).

\section{Results}

Of the 13 observed AQD candidates 6 were confirmed as quasars. A further 4 were identified as stellar contaminants while 3 spectra remained unidentified. This gave an overall success rate for identifications of $77 \%$. Two of the unidentified candidates had low S/N but a contributory factor could be that since FLAIR cannot effectively observe below $4500 \AA$ some of the features visible in the associated objective-prism spectra at these wavelengths were not detected in the FLAIR spectra.

Fig. 1 gives the summed $6 \times 1800 \mathrm{~s}$ FLAIR spectra of the 6 identified quasars after spectral processing (i.e. bias-subtraction, removal of cosmic ray events, wavelength calibration and sky-subtraction). No flux-calibration was possible so the data represent raw total counts strongly affected by the CCD detector sensitivity which falls off sharply below $4500 \AA$. The CCD gain is $\sim 1 e^{-}$per ADU and the readout noise $\sim 10 e^{-}$. Typical $\mathrm{S} / \mathrm{N}$ ratios in the FLAIR quasar spectra continua at $\lambda \sim 5000 \AA$ are $\sim 20$. The presence of two or more identifiable emission lines in 5 of the 6 quasar spectra enable an unambiguous redshift to be determined. The spectra cover $\sim 2250 \AA$ from $\lambda \lambda 3980-6230 \AA$. This compares with $\sim 2000 \AA$ for the objective-prism spectra from $\lambda \lambda 3400-5380 \AA$. The FLAIR wavelength range was chosen to follow as closely as possible the original coverage of the prism spectra so that largely the same spectral features should be detected.

Fig. 2 gives the 6 corresponding low-dispersion objective-prism spectra selected by AQD. It is possible to identify common emission features between the two sets of spectra. A good match between the AQD redshifts and the accurate FLAIR redshifts was achieved for 3 out of the 6 observed quasars. However a poor redshift match was obtained for the two AQD objects Q_6 \& Q-4. Typically only one emission line is seen in the objective-prism spectra of quasars: a redshift can be assigned by guessing its identity (usually guessed to be Ly $\alpha$ since that is most likely) but the process is clearly not ideal. However, even with FLAIR, candidate Q-12 still has only a tentative redshift since only one line is visible in the FLAIR spectrum too.

Table 1 gives the positions, COSMOS magnitudes, estimated B magnitudes, AQD and FLAIR redshifts and identified spectral features for the 6 confirmed quasars.

The COSMOS magnitudes are isophotal and are quoted as magnitudes above the nominal plate limit. In the absence of accurate CCD photometry of the AQD candidates, approximate B magnitudes were obtained using data 
from two published photometric sequences in the adjacent field 295 from Walker (1988) and Alcaino \& Liller (1984). The photographic image diameters of the AQD candidates from the UKST J survey of field 296 were compared with those in the two sequences. (These images were 'boot-strapped' to field 295 using stars in the overlap region between the two fields). An image-diameter versus photometric magnitude calibration plot was then constructed and the AQD candidate image diameters were read off against a least squares fit to the sequence data. This technique has been shown to yield magnitudes accurate to \pm 0.5 and gave AQD candidate magnitudes in the range $B \sim 18.3-18.7$.

Table 1. Summary results for the 6 confirmed AQD quasars

\begin{tabular}{|c|c|c|c|c|c|c|c|}
\hline ID & $R A_{1950}$ & $D E C_{1950}$ & $\mathrm{Bj}$ & $\mathrm{B}$ & $Z_{\mathrm{AQD}}$ & $Z_{\text {FLAIR }}$ & Features \\
\hline Q_4 & 010511 & -403750.2 & -3.12 & 18.3 & 2.32 & 0.331 & FeII, $H \delta$ \\
\hline Q.6 & 013247 & $\begin{array}{llll}-40 & 07 & 25.5\end{array}$ & -2.95 & 18.7 & 2.18 & 0.729 & MgII, FeII, NeV \\
\hline Q_7 & 012742 & $-4002 \quad 25.5$ & -3.07 & 18.6 & 2.16 & 2.158 & CIV, CIII] \\
\hline Q_11 & 011346 & -391501.6 & -3.05 & 18.7 & 2.04 & 2.084 & CIV, CIII] \\
\hline Q_12 & 013219 & -385852.7 & -3.14 & 18.4 & 2.14 & $2.04 ?$ & MgII? \\
\hline-13 & 012550 & -385529.6 & -3.18 & 18.6 & 1.93 & 1.934 & CIV, CIII] \\
\hline
\end{tabular}

Based on the success of this preliminary study a project to utilise FLAIR-II to observe further top-grade AQD candidates in 3 of the 7 SGP AQD fields is underway. The intention is to assist the search for more quasar superstructures.

\subsection{Other work}

Quasar candidates selected by UVX surveys have also been observed with FLAIR to $B<18.5$ but so far with limited success (Goldschmidt et al. 1992). This can be partly attributed to the UVX selection technique which does not necessarily rely on a strong emission line being present. AQD is more successful because strong-lined candidates are naturally favoured by using prism-spectra and the high-grade selection criteria.

\subsection{Recent developments}

An updated FLAIR system, FLAIR-II, was commissioned in 1992. Although the same CCD detector is employed as with PANACHE a completely new spectrograph using all Schmidt optics is now used. This gives greater wavelength coverage at the same resolution whilst allowing up to 92 fibres to be imaged simultaneously on the CCD. This would enable rapid progress with the bright component of AQD surveys and indeed FLAIR-II data in a further AQD survey field has recently been obtained. Full details of the new system can be found in Watson et al. (1993). The current system still however suffers from poor blue sensitivity with a detector quantum efficiency of only $\sim 15 \%$ below $\sim 4300 \AA$ (see Oates 1990). However the planned upgrade to a thinned back-illuminated CCD for FLAIR-II during 1994 is expected to give a 3-fold increase in the $\mathrm{S} / \mathrm{N}$ below $4500 \AA$ or a 1 magnitude gain. 


\section{Conclusions}

FLAIR has been shown to be an effective tool for obtaining simultaneous observations of potentially large numbers of high-grade AQD quasar candidates across the full $40 \mathrm{deg}^{2}$ field of view of the UKST. In this preliminary study out of a total of 13 observed AQD quasar candidates with $B \leq 18.5$, six were confirmed as quasars and four identified as stars. The implementation of a thinned CCD detector on FLAIR would significantly enhance the system's capabilities for performing useful spectroscopic observations of quasars of moderate faintness over large areas of sky.

\section{Acknowledgments}

We gratefully acknowledge the support of the COSMOS team at the Royal Observatory Edinburgh and staff of the UKST in Australian and the UK.

\section{References}

Alcaino G. \& Liller W., 1984, AJ, 89, 814

Clowes R. G., Cooke J. A. \& Beard S. M., 1984, MNRAS, 207, 99

Clowes R. G., 1986, Mitt. Astron. Ges., 67, 174

Clowes R. G. \& Campusano L. E., 1991, MNRAS, 249, 218

Goldschmidt P., Miller L., La Franca F. \& Cristiani S., 1992, MNRAS, 256, 65p MacGillivray H. T. \& Stobie R. S., 1984, Vistas in Astr., 27, 433

Oates A. P., 1990, Proc. SPIE Conf., 1235, 272

Parker Q. A. \& Watson F. G., 1990, A\&AS, 84, 455

Walker A., 1988, PASP, 100, 949

Watson F. G., 1988, ASP Conf. Ser 3, p.125

Watson F. G., Gray P. M., Oates A. P., Lankshear A. \& Dean R. G., 1993, ASP Conf. Ser 37, p.171 